\title{
Indian Literature: The Polyphonic Nature of Deconstructing Myths
}

\section{Samaresh Mondal}

Ph.D Fellow, Indian Comparative literature Department, Assam University (Central), Silchar, India.

Mail Id: mondalsamaresh199@gmail.com | Orcid ID: 0000-0003-4846-4579

\begin{abstract}
In this modernized world, scientific invention may breathe life into poetry and poetry, along with myth will recreate and redefine literature. Like the way, we collect myths; we create and intrude into myths as well. Language and the world consist of myths and finally it gives birth to life.

Etymologically myth came from the Greek word 'muthos' which was later adopted by Latin. Though nowadays the word may signify something else, originally it used to refer to the combination of poetry and music.
\end{abstract}

If we consider myth to be an amalgamation of poetry and music, we can easily state that myth is an imaginative creation of an entire community. This creation is also a process of knowledge production which is explained through the various sensuous colours and forms of different experiences one gathers in one's life. In this process, language as well as the colours and forms change simultaneously and with them, the experiences are re-explained. Thus, the function of myth is to turn experience into knowledge and knowledge into colours and forms. The poet uses myth to achieve a universal truth, which is a general purpose of using myth in any form of verbal arts. Because, it is myth through which the deeper truth can express itself easily and it can expand the horizon beyond the day-to-day notion of beauty. The noted journalist and author, Italo Calvino from Italy opined, Myth is the hidden part of every story, the buried part the region that is still unexplored because there are as yet no words to enable us to get them. Myth is nourished silence as well as by words.

The novel Hanshuli Banker Upakathaby Tara Shankar Bandyopadhyay, which is centred on Indian freedom movement, starts with a particular myth of a whistling sound of semi-divine origin that comes from the forest at night and frightens the kahars. The use of myth along with modernity makes the novel truly polyphonic. The author portrayed the ups and downs of the residents of an insignificant and remote village called 'banshbadi', whose lives revolved around the river kopai. He described how the local beliefs, local myths and folklores were gradually changing and giving way to the modern lores and tales and creating a space for heteroglossia and polyphony. In this context, I have cited few indigenous and foreign authors, not only to strengthen my points, but also to show how myth crosses the spatio-temporal boundaries.

Keywords: Folklore, Myth, Deconstruction, Imagination, Tradition, Modernity, Voices 


\section{ভারতীয় সাহিত্য: মিথ, ঘাত-প্রতিঘাতের বিনির্মাণের বহুস্বর}

সারসংক্ষেপ (Abstract in Bengali)

আজকের এই অত্যাধুনিক বিশ্বে বিজ্ঞানের নিত্য-নতুন আবিষ্কার কবিতাকে সঞ্জীবনী শক্তি দান করে এবং কবিতা মিথের সহযোগিতায় সাহিত্যকে পুনর্নিমাণ ও পুনঃসংজ্ঞায়িত করে। যেমনিভাবে আমরা মিথ সংগ্রহ করি আবার একইভাবে মিথ তৈরি করে আমরা অজান্তেই এর ভেতরে প্রবেশ করি। ভাষা এবং এই জগৎ সংসার মূলতঃ মিথ-সৃষ্ট এবং চূড়ান্তরূপে এদের মিথক্ক্রিয়ায় আমরা জীবনকেই নির্মাণ করি ।মিথনতুনভাবে কথাসাহিত্যকে তৈরি করে কেননা প্রত্যেকটি সৃষ্টি মিথ। আমরা যেমন মিথ সংগ্রহ করি তেমনি আবার মিথ তৈরি এবং মিথের মধ্যে অজ্ঞাতে কখনোবা প্রবেশ করি। ভাষা ও জগৎটা মিথের এবং এই দুইয়ে িলনে জীবন।

মিথ গ্রিক শব্দ মুথোস ( Muthos ) থেকে এসেছে। সম্ভবত আমরা এর আদি অর্থ পরে লাতিন অনুষঙ্গ ' মিথুস (Mythus ) শব্দে হারিয়ে ফেলেছি। কবিতা ও সংগীতের পরিপূর্ণ মিলনের এক রহস্য ও জাদু এর মধ্যে নিহিত।

আমরা যদি মিথকে কবিতা ও সংগীতের মিলন রূপে কল্পনা করি, তাহলে এ সত্য আমাদের স্বীকার করতে বাধা নেই, মিথ হচ্ছে একটি সমগ্র জাতির কল্পসৃষ্টি। এই কল্পসৃষ্টির মধ্যে মানুযের জ্ঞান কাজ করে, জ্ঞানকে সে ব্যাখ্যা করে সতেজ সজীবতায়, ছবির রূপে রঙে বেদনায় সংবেদনার অনুভূতিতে। অভিজ্ঞতায় যে জ্ঞান পেয়েছে, সেই জ্ঞানকে এমনিভাবে ব্যাখ্যা করে। এক্ষেত্রে ভাষা অনবরত পরিবর্তিত হয়, ভাষার পরিবর্তনের সঙ্গে ছবি ও রঙ পাল্টায়, ছবি ও রঙের সঙ্গে সুর ও ব্যাখ্যা কিছু নতুন রূপ পায়।অভিজ্ঞতাকে জ্ঞানে, জ্ঞানকে ছবির মধ্য দিয়ে আর এক অভিজ্ঞতায় ধাক্কা দেওয়াই মিথের কাজ। সুতরাং কবি যখন মিথ ব্যাবহার করেন, তখন এক সর্বজনীন সত্যকে লাভ করতে চান, এমনকি কথাসাহিত্যের মধ্যেও মিথ সর্বজনীনতা প্রকাশ পায়। কেননা মিথের মধ্যে গভীর সত্য প্রকাশিত হতে পারে সহজভাবে। এমনকি সৌন্দর্য ছাড়িয়ে একটা বিশাল উপলব্ধির আকুলতা এই মিথ তৈরি করে। এই কারণেই মিথ কাব্যিক দর্শন হয়ে ওঠে। ইতালির বিশিষ্ট সাংবাদিক, কথাসাহিত্যিক ইটালো ক্যালভিনো ( ১৫ অক্টো ১৯২৩ -- ১৯ লেপ্টেম্বর ১৯৮৪৪) মিথ সম্পর্কে একটি উক্তি করেছিলেন -- Myth is the hidden part of every story, the buried part the region that is still unexplored because there are as yet no words to enable us to get them. Myth is nourished by silence as well as by words.

তারাশঙ্করের ' হাঁসুলী বাঁকের উপকথা '( ১৯৪৭) স্বাধীনতার প্রেক্ষাপট, শুরুতেই মিথের আবির্ভাব। রাতে ঘন জঙ্গলে শিস কার -- দেবতা, যক্ষ কি রক্ষ, কার, কাহারেরা সন্ত্রস্ত। মিথ ও আধুনিকতায় উপন্যাসটি হয়ে উঠেছে বহুকৌণিকের কণ্ঠস্বর। বীরভূনের একটি নগন্য গ্রাম ' বাঁশবাদি ' কে কেন্দ্র করে নিম্নবর্গ মানুষদের নিয়ে লেখক ভাঙা গড়ার চিত্র অঙ্কন করেছেন। উপন্যালে দেখা যায়, কোপাই নদীকে কেন্দ্র করে যে সব সম্প্রদায়ের মানুষের বসবাস তাদের যে লোকবিশ্বাস, পুরাণ, মিথ ছিল সেখান থেকে বেরিয়ে এসে নতুন বিশ্বাস আঁকড়ে ধরছে এবং বহুকৌণিকের গমক সৃষ্টি হয়েছে। আলোচনার সুবিধার্থে দেশি ও বিদেশি সাহিত্যের প্রসঙ্গ এনেছি সেটা কেবলমাত্র আমার বক্তব্যকে জোরদার করার জন্য নয় বরং ভাঙা গড়ার বিনির্মাণের জগতের ভিন্ন সময়ের স্বর কীভাবে উঠে এসেছে সেই বিষয়ে আলোকপাত করার চেষ্টা করেছি।

সূচক শব্দ (Keywords in Bengali): োকবিশ্বাস, মিথ, ভাঙা গড়া, কল্পনা, জগৎ, সংস্কার, আধুনিকতা, উপাদান, কণ্ঠস্বর।

89

Litinfinite Journal is Indexed By MLA Directory Of Periodicals \& MLA International Bibliography, EBSCO, ProQuest, SCILIT, Ulrichsweb \& Ulrich's Periodicals Directory, ICI World Of Journals, J-Gate, JISC-Sherpa Romeo, DRJI, EuroPub \& Other Major Indexing Services. (This Open Access article is published under a Creative Commons Attribution Non-Commercial 4.0 International License.) 


\section{ভূমিকা}

আমরা यদি বিশ্বসাহিত্যের মহাকাব্য থেকে ওরু করে মহৎ যেসব সাহিত্য রয়েছে তার দিকে তাকাই, দেখব যে, মহৎ সাহিত্য থেকে নতুন নতুন বর্গের( Genre ), নতুন নতুন ঘরানা, নতুন নতুন বাঁকের সৃষ্টি হয়েছে। ঠিক সেভাবেই ভারতের দুটি মহাকাব্য রামায়ণ ও মহাভারত থেকে মিথ ও বাস্তবের সঙ্গে সঙ্গে বর্গের (Genre) সূত্রপাত হয়েছে। আর এই বর্গ তো পরিবর্তনের দারমুখি কেননা এগুলি তো ইতিহাসগত আশ্রয়। রামায়ণ প্রসঙ্গে বলা যায়, রাম সীতার ক্ষেত্রে মূল মিথ হল লোক প্রচলিত বিষয় এবং আর্যভাবী জনতার বিস্তারের সঙ্গে সঙ্গে স্থানিক ও সামাজিক পরিস্থিতি অনুযায়ী কালক্রমে বিবরণগত ও ব্যাখ্যাগত পার্থক্য আরোপিত হুত থাকে। মনে করা যেতে পারে, বাল্মীকি সে সময় ব্রাঙ্ষণ্যবাদী আর্য এলাকা মধ্যদেশে প্রচলিত এমনই কোনো একটি রূপক কাহিনি আশ্রয় করে সংস্কৃত ভাযায় রামায়ণ রচনা। রাম সীতা মিথের বিভিন্ন আখ্যান নিশয়ই অন্যান্য স্থানে সেই সময়ে প্রচলিত ছিল। যেমন দশরথ জাতক ও অন্য কোনো জাতকের পাঠভেদের ব্যাখ্যাই তাই। আবার দেখা যায়, অশ্বঘোমের রচনায় রাম সীতা মিথের যে উল্লেখ রয়েছে এবং পরবর্তীকালেও দেশান্তরে এই মিথের যে অন্য কাহিনি প্রচলিত তারও ব্যাখ্যা একই।

মহাভারতের ক্ষেত্রে ভারতযুদ্ধ বা কুরুক্ষেত্র যুদ্ধের মূল মিথটি আপেক্ষিক বিচারে অপরিবর্তিত বলা যায়। পরস্পর মৌলিক পার্থক্যযুক্ত বর্ণনাগতপাঠভ্দএকক্ষত্রে বিরল,যেটুকু পার্থক্য আছে তাতে যুযুধান উপজাতিগুলির অথবা যুদ্ধক্ষেত্রের প্রধান কুশীলবদের ভূমিকায় বিশেষ পার্থক্য নেই। এবং পারস্পরিক অবস্থার বর্ণনাও প্রায় একই।অর্থাৎ এই বিষয়ে রামায়ণ আর মহাভারতের মূল মিথ দুটির গুণগত পার্থক্যও তাৎপর্যপূর্ণ। তবে একথাও সত্য যুগ ও স্থানের পরিবর্তনের সঙ্গে সন্গে ব্যাসগণের কাব্যপ্রয়ালে শেমের কাহিনিত ব্যাপক এবং বিচিত্র সংযোজন ও সমাবেশ ঘটেছে। এগুলি মূলত পৃথক, যোগসুত্রহীন বা অতি শিথিল সংরোগ মিথ, উপকথা, আখ্যান, উপাখ্যান, কল্পনা, নীতিশিক্ষা এবং ধর্মের পাদদ্দেশ কেন্দ্রীয় মিথ কুরুক্ষেত্র যুদ্ধকে ঘিরে রচিত।

তারাশঙ্করের 'হাঁসুলী বাঁকের উপকথা'(১৯৪৭) স্বাধীনতার প্রেক্ষাপট, শুরুতেই মিথের আনাগোনা, মিথ ও আধুনিকতায় উপন্যাসটি হয়ে উঠেছে অনন্য। দ্বিতীয় বিশ্বযুদ্ধের কিছু আগের সময়ে বীরভূমের একটি নগণ্য

90

Litinfinite Journal is Indexed By MLA Directory Of Periodicals \& MLA International Bibliography, EBSCO, ProQuest, SCILIT, Ulrichsweb \& Ulrich's Periodicals Directory, ICI World Of Journals, J-Gate, JISC-Sherpa Romeo, DRJI, EuroPub \& Other Major Indexing Services. (This Open Access article is published under a Creative Commons Attribution Non-Commercial 4.0 International License.) 
গ্রাম বাঁশবাদি। তার পাশ দিয়ে বয়ে যায় কোপাই। তারাশঙ্করের লেখায় একটা স্বাভাবিক ও স্বত:স্ফূর্ত বিচরণ ক্ষেত্র হল রাঢ়ভূমি।এক্ষেত্রে প্রেমেন্দ্র মিত্রের উক্তি স্মরণ করা যেতে পারে -- 'বাংলাদেশের ভৌগোলিক মানচিত্রে রাঢ়দেশের স্থান আছে ও থাকবে। কিন্তু সাহিত্যে তাকে অবিনশ্বরতা দিয়ে গেছেন তারাশংকর'১( তারাশঙ্কর সংখ্যা, কোরক সাহিত্য পত্রিকা, ৫৩)। এটি একটি আঞ্চলিক উপন্যাস। আঞ্চলিকতার পটে সর্বজনীনতার বৃহত্তর ব্যঞ্জনা যে তারাশঙ্করের সাহিত্যে মূল কথা, লেটাও প্রেমেন্দ্র মিত্র সুন্দরভাবে বলেছেন -- 'প্রতিমা নিঁখুত করে অনেকেই গড়তে পারে, কিন্তু তার চক্ষুদানই হল আসল। শিল্পীর সেইটাই সবচেয়ে বড় পরীক্ষা। চক্ষুদান যথার্থ যদি না হয় তাহলে প্রতিমা গড়ার সব বাহাদূরী বরবাদ হয়ে তা মাটির পিন্ডই হয়ে থাকে মাত্র। সেই। চক্ষুদান 'এর ক্ষমতা যদি না থাকত, তাহলে রাঢ় দেশ নিয়ে তারাশঙ্কর যত কিছু লিখে গেছেন তা একটি বিশেষ অঞ্চল আর সময়ের দলিলধর্মী বিবরণের বেশি মূল্য ভাবীকালে পেত না' ${ }^{\prime}$ (তারাশঙ্করসংখ্যা,৫৩)।

এই ভৌগলিক পটভূমিতে লেখক দুটি সম্প্রদায়কে রাখছেন -- গাঁয়ের বাবুমশাইরা এবং গ্রামের চাষি, সদগোপ, নাপিত, কোল প্রভৃতি সভ্য সম্প্রদায়। আর কাহারপাড়া যাদের নিম্নবর্গ বলা যেতে পারে। উপন্যাসে অসংখ্য চরিত্র আছে যেমন - সূচাঁদ, বনওয়ারী, করালী, প্রহ্লাদ, গোপীচাঁদ, রতন, অমন, পরম, পরমের স্ত্রী কালোশশী ইত্যাদি -- এদের প্রত্যেকের কিছু না কিছু ভূমিকা আছে। বনওয়ারী ও করালী উপন্যাসে মুখ্য ভূমিকা পালন করেছে। এরা নানারকম কুসংস্কার মানে। সমাজে প্রচলিত রয়েছে যে, সংসারে লক্ষ্মী এনেই নাকি সব ঠিক হয়ে যায়, যদিও এটা কাহারেরা মানে। বাবাঠাকুরের বাহন অর্থাৎ চন্দ্রবোড়া সাপ উঠোনে পড়ে আছে, করালী দেখেই বুক চাপড়ে কেঁদে ওঠে । তাই সে বলে --' ওগো বাবা ঠাকুর গো ! ওরে আমার বাহন রে ! ওরে কি হবে রে ! হায় মা রে !৩ ( বন্দ্যো,৩৫)। উপন্যাসে লক্ষনীয়, মাইতো ঘোষকে চন্দনপুর কারখানা থেকে তাড়িয়ে দিয়েছে অথচ সেই কারখানা সঙ্গে তার মায়ের ইতিহাস জড়িয়ে রয়েছে। তিন বছর আগে করালীর বাবা যখন মারা যায় তখন করালী ছেলে মানুষ সেই তখন থেকে হাঁসুলী বাঁকের উপকথা এক টুকরো গল্প -- গোটা পাঁচালীর মধ্যে কয়েকটা ছড়া। এসব অনেক দিনের পুরনো গল্প ; এখনও চলে আসছে এবং চলছেও। সেই সব ছড়া বলতে বনওয়ারীর মতো মাতব্বরেরা লজ্জা পায়। তাই সূচাঁদ বলে --' আ: তায় আবার লাজ কিসের? বলে সেই বেগুনে কেন খাড়া? না বংশাবলীর ধারা' ${ }^{8}$ (বন্দ্যো:,৩৮)।

তারাশঙ্কর অভিজ্ঞতার শিল্পী। সেই অভিজ্ঞতা কাজে লাগিয়ে তিনি একেবারে প্রত্যন্ত 'রাঢ়' অঞ্চলকে বেছে নিয়েছিলেন। তিনি সেই অঞ্চলের মানুষ হওয়ায় নিজ ভূমিকে দেখেছেন, চিনেছেন, ভালোবেসেছেন। তাই 
বীরভূন্ের 'রাঢ়' ভূখণ্ডটি উপন্যাসের কেন্দ্রবিন্দু -- তার আদিবাসী কাহার, বেদে, ডোম, বীরবংশী, বাউরী,বাগদী, সাঁওতাল, চন্ডাল, ডাইনী প্রভৃতি নিম্নবর্গের মানুষ গুলির জীবনচিত্র অঙ্কন করেছেন। উপন্যাসে যেসব মানুষের কথা বলা হয়েছে তারা সমাজে পিছিয়ে পড়া মানুষ, আধুনিক 'সাবলটার্ন' তত্ত্ব অনুযায়ী নিম্নবর্গের আওতায় চলে আসে। 'সাবলটার্ন'এর বাংলা প্রতিশব্দ 'নিম্নবর্গ'। ১৯৮০ দশকে ' সাবলটার্ন ' শব্দটি ভারতীয় ইতিহাস চিন্তায় একটি মুখ্য স্থান অধিকার করে নিয়েছে। নিন্মবর্গের বৈশিষ্ট্য হতে পারে --
১) সমাজে পিছিয়ে পড়া মানুষ।
২) যাদেরকে সবসময় কোনো ধনী বা প্রভাবশালী ব্যক্তি দমিয়ে রাখতে চায়।
৩) এদের কোনো প্রতিবাদের কণ্ঠস্বর থাকে না।

পঞ্চাশের দশকে ফরাসি চিন্তাবিদ রোলাঁ বার্ত তাঁর 'মিথোলজি' গ্রন্থটিতে মিথ তন্ত্রের সূক্ম বিচার বিশ্লেষণ করেছেন। তাঁর মতে, মিথ সমাজকে বেঁধে রেখে সামাজিক ঐক্য ও পরম্পরাকে ধরে রাখে।সেই সঙ্গে অতীতের পুরাণ, বিশ্বাস, সংস্কার ইত্যাদিকে স্বতন্ত্রভাবে এগিয়ে নিয়ে যায়। সমাজের মধ্যে মিথের ভাঙা গড়ার প্রক্রিয়া চলতে থাকে। মিথ সব সময় পরিবর্তনের কারণ মিথ কখনো নির্দিষ্ট জায়গায় থেনে থাকে না।

'হাঁসুলী বাঁকের উপকথা' উপন্যাসে ঔপন্যাসিক নিজেই লিখেছেন -- "হাঁসুলী বাঁকের দেশ কড়া ধাতের মাটির দেশ। এদেশের নদীর চেয়ে মাটির সঙ্গে মানুষের লড়াই বেশি। 'খরা' অর্থাৎ প্রখর গ্রীষ্ম উঠলে নদী শুকিয়ে মরুভূমি হয়ে যায়, ধূ ধূ করে বালি --একপাণে মাত্র একহাঁটু গভীর জল কোনো মতে বয়ে যায়।... মাটি তখন হয়ে ওঠে পাষাণ। ঘাস যায় শুকিয়ে,মাটি গরম হয়ে ওঠে আগুনে পোড়া লোহার মত; কোদাল কি টামনায় কাটে না, কোপ দিলে কোদাল টামনারই ধার ব্রেকে যায়; গাঁইতির মত যে যন্ত্র লে দিয়ে কোপ দিলে তবে খানিকটা কাটে কিন্তু প্রতি কোপে আগুনের ফুলকি ছিটকে পড়ে। খাল বিল পুকুর দীঘি চৌচির হয়ে ফেটে যায়। বীরভূনের এই মাটির পরিচয় তারাশঙ্করের অভিজ্ঞতায় পাওয়া যায় পরবর্তীকালেও। 'আমার কালের কথা' য় নিজের সূতিকাগৃহ প্রসঙ্গে তারাশঙ্কর লিখেছেন, মাটির মেঝে, শক্ত পাথুরে রাঙ্গামাটির দেওয়াল দিয়ে গড়া উত্তর দুয়ারী কোঠাঘর আজও অটুট আছে।... ঘরখানির সামান্য পরিবর্তনের জন্য বছর কয়েক আগে খানিকটা দেওয়াল ভাঙার প্রয়োজন হয়েছিল, কোদাল, টামনা, শাবল হার মানলে, শেযে গাঁইতি আনা হল; 
দেওয়াল ভাঙল বটে কিন্তু সেদিন যে আগুনের ফুলকি ছড়িয়েছিল গাঁইতির আঘাতে, তা আজও আমার চোখে ভাসছে "৫ ( মুখো:,१৪ )

মিথ তো বহুস্তরীয় ভাবনার সংমিশ্রণ। যদি আফ্রিকার সাহিত্যে মিথের কথা ভাবি দেখব যে, লেখ্যচর্চার আগে এই মহাদেশের সাংস্কৃতিক ঐতিহ্যের একমাত্র উপায় ছিল বংশানুক্রমে মৌখিকভাবে বয়ানকে ধরে রাখা। তাদের ইথিওনিয়া ও লাইবেনিয়া নানে দুটি জনগোষ্ঠীর ছিল। এই দুটি ছাড়া সাহারা উপান্তের আফ্রিকায় ইউরোপীয় আসার আগে লেখার ছিল না। সেজন্য তাদের পুরা আখ্যান একটি নির্দিষ্ট অঞ্চলে সীমাবদ্ধ থেকে যায়। সময়ের সঙ্গে সঙ্গে বদলে যায় তাদের আর্থ সামাজিক প্রেক্ষাপট। বংশানুক্রমিক মৌখিকভাবে যেটা প্রচলন ছিল সেখান থেকে সরে আসে। আসলে আফ্রিকায় অঞ্চল ভিত্তিক কত্তম বা গোষ্ঠীগুলির মধ্যে পারস্পরিক দূরত্ব থাকায় এবং বিজ্ঞানের প্রসার না থাকার ফলে আফ্রিকা ছিল পুরো আখ্যানের সম্ভাবনাপূর্ণ ভূমি।

ঔপনিবেশিক শাসনে নিজেদের সংস্কৃতি কৃষ্টি প্রজাতিকে গৌরবান্বিত করার লক্ষ্যে উচ্চবর্ণ সম্প্রদায়ের সাদা চামড়ার মানুষেরা হাজারবার একথাই বলে এসেছে যে, পাশ্চাত্য সংস্পর্শের আগেই এই মহাদেশের কোনো সংস্কৃতি ছিল না। এই অবমাননাকর কথা স্বদেশবাসীর মনে তীব্র প্রতিত্রিয়া তৈরি করে, ফলস্বরূপ সাহিত্যিকরা প্রমাণ করেন যে, তাদের মাতৃ পিতৃভূমি কোনোদিনই সংস্কৃতিহীন ছিল না। সেজন্য চিনুয়া আচেবে প্রত্যুত্তরে জানালেন --" যুরোপীয়দের থেকেই কৃষ্টির কথা প্রথমবার জানছে আফ্রিকা তা নয়। অপরিচিতের হাতে দেশ মানুষের অপমান। অবমাননা থেকে মনুষ্যত্ব, মানবিকতাকে ফিরিয়ে আনলেন রচয়িতারা তাঁদের সৃষ্টি কৃতিতে। ঔপনিবেশিক শাসনে চাপা পড়ে যাওয়া স্বদেশের ইতিহাস উদঘাটনে দেশবাসীর সঙ্গে বৃত হলেন লেখক শিল্পীরা। স্ট্যানলেন সমকাঙ্গে ' অন ট্রায়াল ফর মাই কান্ট্রি । উপন্যাসে সেসিল রোডেস এর ঔপনিবেশিকরনের ঐতিহাসিক আখ্যান শোনালেন আমাদের " ৬ ( সেন ওসিংহ, b-১)।

'হাঁসুলী বাঁকের উপকথা' উপন্যাস লেখক খুব সচেতন দৃষ্টিভঙ্গিতে শিল্পরূপ নির্মাণ করেছেন। উৎসর্গ পত্রে কবিশেখর কালিদাস রায়ের উদ্দেশ্যে লিখেছেন -- সেখানকার মাটি মানুষ তাদের অপভ্রংশ ভাষা - আপনার সুপরিচিত। রাঢ় অঞ্চলের রাঢ়ী উপভাষা উপন্যাসে অনেকাংশেই জুড়ে আছে। হাঁসুলী বাঁকের মানুলেরা বলে ক) 'রঙ' কে বলে 'অঙ', 'রাম' কে বলে 'আম', 'রজনী' কে বলে 'অজনী', 'রীতিকরণ' কে বলে 'ইতকরণ' খ) 'অ্যামোনিয়া' কে বলে 'আলুমিনি', 'অ্যালুমিনিয়াম' কে বলে 'এনামিলি' কাহারেরা আবার ভীষণ রসিকতার 
মানুষ। যখন কোনো মনিব বেশি জট পাকায় তখন তাকে ওরা Code নাম দেয় যেমন, পাকু মণ্ডল, মোটা হলে ' হেনো মন্ডল। এমনকি তারা যখন কোনো কিছুতে প্রশংসা করে তখন বলে 'বাহা বাহা'।

কাহারদের মধ্যে যখন ঝগড়া হয় তখন একদিনে থামে না দিনের পর দিন চলতে থাকে। তাদের মধ্যে গালিগালাজ, ঝগড়াঝাটি নিত্যদিনের ব্যাপার, এই সংস্কৃতি পুরুষানুক্রনে চলে আসছে। সূচাঁদ যেহেতু বয়সে সর্বশ্রেষ্ঠা তাই সে একটু মন্ত্র জপ জানে। নয়ান হাঁপানিতে ভুগছে, সূচাঁদ তাকে একটা মাদুলি দেবে যাতে তা থেকে মুক্ত হয়। এই বিশ্বাস বর্তমান সমাজ ব্যবস্থা রয়েছে কিন্ত্ত আধুনিক সভ্যতার কাছাকাছি এসে মিথেত জগতের প্রতিরূপ তৈরি করার ক্ষমতা ক্রমশ হ্রাস পায়। হাঁসুলী বাঁকের দিন চলে গেলে রাত্রি নেমে আসে এই রাত্রির সঙ্গে জাঙল চন্ননপুরের রাত্রি অনেক তফাৎ। হাঁসুলী বাঁকের কাহারেরা কেরোসিনের ডিবা জেবে কর্তা ঠাকুরের নাম নিয়ে জটলা পাকিয়ে বসে। ছেলে ছোকরারা ঢোল বাজিয়ে কখনও ধর্মরাজের বোলান, কখনও গায় মনসার গান পাঁচালি, কার্তিক থেকে মাঘ ফাল্গুন পর্যন্ত শীত -- তখন গান বাজনার আসর ঝিমিয়ে আসে কেননা ধান কাটার ফসল তোলার সময়। চৈত্র মাসে নতুন করে ঘেঁটুগান, সংক্রান্তির শেমের দিকে বলে গাজন, বোলানের গানের পালা। কাহারদের কাছে অলৌকিক দেবদেবীর কথাই হয়ে উঠেছে একমাত্র সত্য -- এটা যুগ যুগ ধরে চলে আসায় মিথ বিশ্বাসে পরিণত হয়েছে। আটপৌরেদের লোকায়ত ঘেঁটুগানের জোর সত্যিই বেশি। সেজন্য বনওয়ারী দাঁড়িয়ে শোনে -

\section{"হায় কলিকালে, কতই দেখালে}

দেবতার বাহন পুড়ে ম' ল অকালে, তাও মারলে রাখালে।

ও তার বিচার হুল না বাবা, তুমি বিচার কর।

অতি বড় বাড়িল যারা, তাদের ভেঙে পাড়ো" প( বন্দ্যো:, b-৯ )

কাহারেরা এও বিশ্বাস করে যে, প্রথম প্রথম যখন উঠবে তার ভোগ দিতে হবে বাবাঠাকুরের থানে। যেখানে থাকবে মুগসিদ্ধ, বরবটি সিদ্ধ আর এক বোতল মদ। কোপাই নদী এবং হাঁসুলী বাঁকের প্রকৃতির সঙ্গে অত্যন্ত শ্রেণির এতটাই একাত্মতা। কাহারেরা তাদের প্রাচীন সংস্কার ও বিশ্বাস নিয়েই পড়ে আছে। এ জগৎ, আলাদা জগৎ। তাদের ঐতিহ্য, তাদের অন্ধবিশ্বাস, তাদের রীতিনীতি আদব কায়দা, তাদের সংস্কার নিয়ে গড়া এই জগৎ; যেখানে আধুনিক সভ্যতার আলো এসে পৌঁছায়নি। এই আদিম পদ্ধতিতে জীবন ধারণকারী অন্ত্যজ 
কাহার জীবনেও একদিন সভ্যতার আলো এলে পৌঁছাল। আলো এনে দিল কাহারদের ছেলে তথা আধুনিকতার প্রতীক করালী।

হাঁসুলী বাঁকের উপকথা, কাহাদের জীবনকথা। সেই জীবন -- মানুষ, প্রকৃতি, বিধির বিধান, আচার-বিচার, ধর্মসংস্কার, মিথ দিয়ে গোড়া। এই উপন্যাস উপকথা, না ইতিহাস সেই নিয়ে দ্বন্দ্ব আছে ---"ইহা ইতিহাস নহে, উপকথা। ইহার জীবনযাত্রা অতিপ্রাকৃতের ঘন কুহেলিকামন্ডিত ; পৌরাণিক কল্পনা, অলৌকিক সংস্কার ও বিশ্বাস, প্রাচীন কিংবদন্তি ও আখ্যান, সদ্য অতীতের ঘটনা প্রতিফলিত জীবন দর্শন -- এ সমস্তই প্রাত্যহিক জীবনের রন্ধ্রেরন্ধ্রে, গভীরভাবে অনুপ্রবিষ্ট । হাঁসুলী বাঁকের কাহারদের জীবন দর্শন অপরিবর্তনীয় ভাবে স্থিরীকৃত -- কাহারদের জীবনের যাহা কিছু ব্যতিক্রম ও বিপর্যয়, যাহা কিছু আকস্মিক ও অসাধারণ সবই দেবলীলা, অদৃশ্য -- শক্তির দুর্বোধ্য অভিপ্রায় হইতে উৎক্ষিপ্ত।. . . অশীতিপর বৃদ্ধা সূচাঁদ এই দৈবশক্তির অধিকারিণী ও ব্যাখ্যাত্রী; হাঁসুলী বাঁকের জন্ম বৃত্তান্ত, উহার অতীত কাহিনি, উহার কৈকোর ও প্রথম যৌবনের সমস্ত উদ্ভট কল্পনাও অতিপ্রাকৃত অভিজ্ঞতা পারলৌকিক জগৎ হইতে অভ্যাগত প্রতিটি ধ্বনিও স্পর্শ, দেবতার রোষ ও প্রাসাদের প্রতিটি নিদর্শন তাহার স্মৃতির ঐতিহাসিক আঁধারে অখন্ড সমগ্রতায় ও প্রথম অনুভূতি গাঢ় বর্ণলোপ অবিস্মরণীয়ভাবে রক্ষিত। সেই এই সম্প্রদায়ের Prophet বা আধ্যাত্মলোকের সহিত যোগাযোগ রক্ষার সেতু "b- ( সিংহ ও সেন, ১৫১)।

করালী, কাহারদের ধর্ম সংস্কারে আঘাত এনেছে, সংস্কৃতিতে এনেছে পরিবর্তন। োষকের অন্যায়ের প্রতিবাদ করেছে সে। উপকথায়, ইতিহাসে যুগান্তকারী পরিবর্তন ঘটেছে। কাহার সংস্কৃতিতেও পরিবর্তন । করালী পাখির সঙ্গে 'সাঙা' এমনই পরিবর্তন সূচিত করল। কাহারপাড়া উপকথায় বরের সাজ সজ্জায় করালী কলিযুগে এনেছে। প্রবীনদের মতে, 'এতটা ভালো নয়'। সম্ভবত বনোওয়ারী এর কোনো বিহিত করেনি, কালোশশী ঘটিত দুর্বলতার জন্য বরং নয়ানের ঘর ভেঙে কোরালীর ঘর বসানোর জন্য, সে সমালোচিত হয়েছে। তবে সূচাঁদের আক্ষেপ নয়ানের মায়ের গালিগালাজ শুনে, তার মনে হয় - করালী কর্তার বাহনটিকে মেরে অন্যায় করেছে -- আর তখনই তার হয়ে কর্তার কাছে ক্ষমা প্রার্থনা করে কিন্তু শাস্তি দিতে পারে না। অদ্ভুত প্রক্রিয়ায় সে বুঝতে পারে, কর্তা তার উপর ক্রুব্ধ হননি। নিশ্চিত মনে বিয়ের আসরে এসে দেখে, সেকালের বুড়ি সূচাঁদ, একালের বিয়ের আসর জমিয়েছে। 
এই সংস্কার গ্রাম্য মানুষের মনে বাসা বেঁধেছিল। সুতরাং শিক্ষিত কাহারদের মধ্যে এই সংস্কার খুব স্বাভাবিকভাবেই প্রতিফলিত হতে দেখা যায়। সূচাঁদের মুখে শোনা যায় -- হাঁসুলী বাঁকের লেকালের ভৌতিক লোকের ইতিকথা।

কিন্তু যখন তারা সেকালকে অতিক্রম করে একাল অর্থাৎ আধুনিক যুগে আসার সঙ্গে সঙ্গে তাদের সব কিছুতেই পরিবর্তনের রূপ লক্ষ্য করা যায়। এই যুগ পরিবর্তনের ফলে অন্ত্যজ কাহার কুলের প্রাচীনা সূচাঁদও যেন সন্দিহান হয়ে ওঠে। তার বিশ্বাস কিছু কমেনি কিন্তু এ যুগের মানুষের মনে যে সন্দেহ ঘনীভূত -- তা সূচাঁদের বক্তব্যে প্রমাণিত -- মানুষের আধ্যাত্মিক জীবনের মতো এতেও যেন পরিবর্তন ঘটেছে। এখন ভূত হলে চন্ননপুরের ছোকরাবাবরা বন্দুক নিয়ে পাহারা দিয়ে পরীক্ষা করে দেখতে আসবে।

অন্ত্যজ শ্রেণির মানুষদের নিয়ে লেখা উপন্যাসগুলির মধ্যে 'তিতাস একটি নদীর নাম' উপন্যাসটি একটু স্বাতন্ত্র দাবি করে। বাংলা সাহিত্যে অন্ত্যজ ধীবর মালোদের নিয়ে যে সকল উপন্যাস লেখা হয়েছে তার মধ্যে 'তিতাস একটি নদীর নাম' উপন্যাসটি অন্যতম। হাঁসুলী বাঁকে যেমন, তিতাসেও একটি বিশেষ অঞ্চনের জীবন চিত্র ধরা পড়ে। মানুষের বিশ্বাস, আচার-ব্যবহার, ঐতিহ্য এগুলি তো একটা সময় মিথ বিশ্বাসে পরিণত হয়। সময়ের সঙ্গে সঙ্গে মিথ বিশ্বাসেরও পরিবর্তন ঘটে। মালোদের তিতাসকে নিয়ে যে বিশ্বাসে অটল বাঁধতো লেই বিশ্বাসেও ফাটল ধরে এবং তারা নতুন কোনো বিশ্বাসকে আঁকড়ে ধরতে চাইছে যেখানে গেলে তারা বেঁচে থাকার অধিকার পায়।

গ্রিক পুরাণে মেটিস নামে এক দেবীর উল্লেখ পাওয়া যায়। তার কাহিনি সংক্ষিপ্ত এবং করুণ। সে জিয়ুস এর প্রথম স্ত্রী এবং তার কাহিনী পরিসমাপ্তি ঘটে যখন জিয়ুস তাকে উদরস্থ করে। অন্যদিকে 'মেটিস' শব্দটি একটি সাধারণ বিশেষ্যপদও বটে, এবং তার ধর্ম বুদ্ধিমত্তা অথবা বৈষয়িক জ্ঞান। ব্যবহারিক জীবনে এর মূল্য অপরিসীম। জীবনের ভিন্নধর্মী ক্রিয়াকর্মে 'মেটিস' দরকার হয়। কোনো সময় নাবিকের বিদ্যায় কারিগরের শিল্পে অথবা যুদ্ধ চালনায় তার স্থান যেমন স্বীকৃত, তেমন ছলচাতুরিতেও। কিন্তু জিয়ুস দ্বারা উদরস্থ দেবীর সঙ্গে জীবনের অনিশ্চয়তার মধ্যে সাফল্য অর্জন করার প্রাজ্ঞতার এক নিবিড় যোগ আছে। যেহেতু জিয়ুস মেটিসকে হজম করে ফেলেছেন সেহেতু লে দেব এবং পশুদের মধ্যে সবচেয়ে বুদ্ধিমান এবং দূরদর্শী। প্রসঙ্গক্রমে বলা যায়, খুব সঙ্গত কারণেই বীরেন্দ্র চক্রবর্তী বলেছেন -- "একটি বই এক বা একাধিক ব্যক্তির কাছে ভিন্নপাঠ হয়ে ওঠে যে অর্থে; এবং ইতিহাসও তো আসলে his knowledge of history এমন নয় যেন 
ইতিহাস নামক নির্দিষ্ট কোনো বিদ্যা চিরতরে নির্ধারিত হয়ে আছে কোথাও, মুখস্থ করে নিলেই তা স্বতই পরিণত হয়ে যাবে জ্ঞানে। . . . বিভিন্ন অবস্থান সম্তব যা বিভিন্ন দিককে উন্মোচিত করে"ম ( সিংহওস্সন, ১৫)। স্বাভাবিকভাবেই প্রশ্ন ওঠে, মিথলজি কি ইতিহাসের মতোই মানববিদ্যা যা ব্যক্তি বিশেষে পরিবর্তনশীল? আমার মনে হয়, সমাজতত্ত্ব, রাজনীতি ইতিহাস ও মিথলজি এক ধরণের সাপির হ্বর্ফ এর (Sapir Whorf) 'ভাষিক আপেক্ষিকতা' তত্ত্বের ( Theory of Linguistic relativity ) মতো কোনো তত্ত্বের আওতায় পড়ে।

একটি শিশ্রের মধ্যে অনুভূতির বিস্ময়ে যে গল্প কাহিনি তৈরি হয় তার মধ্যে আদর্শ সত্য আছে; কাব্যিক সত্যিই দার্শনিক সত্যিই। यিনি মিথ তৈরি করেন কবিতায়, গল্প বা কাহিনিতেই, আর যে পড়ে, এই দুইক্রের মধ্যে একজন নান্দনিক অভিজ্ঞতাকে প্রকাশ করে, আর একজন অভিজ্ঞতাকে উপলব্ধি করে আনন্দ পায়। আদর্শ সত্য একমাত্র জ্ঞানের পক্ষেই পাওয়া সম্তব। এই জ্ঞানে মনের সব কিছুই আছে একসন্সে জড়িত; ইনস্টিংট্ট স্বজ্ঞাবোধে মিলিত হয়ে এক হয়ে উপরে উঠতে থাকে, শ্ু বুদ্ধি দিয়ে জ্ঞানের তত্ত্ব প্রতিষ্ঠা নয়।

কাব্যকে এই হিসেবে গণ্য করলে সব কাব্যই মিথ প্রাণিত, এবং মিথ প্রাণিত কবিতাই কাব্যিক দর্শনের স্থান পেতে পারে। তা লে আট পংক্তির রবীদ্দ্রনাথের ক্ষুদ্র গানের রূপ হোক অথবা পাঁচশো পৃষ্ঠার কাহিনি হোক, প্রত্যেকের মধ্যেই মিথ গড়ে ওঠে। মিথের সন্দে জড়িয়ে থাকে রূপকথার স্বপ্ন এবং লেজেজ্ডের কাহিনি ও ইতিহাসমুখিতা এই তিনটির উৎপত্তি ফ্যান্টাসি থেকে। ফ্যান্টাসি ব্যক্তিগত স্বপ্নের ছবি। স্বপ্নের ছবি বলেই বাস্তব জগৎ থেকে পালিয়ে যায়, কখনো হারিয়ে যায়। ফ্যান্টাসির স্বপ্ন যখন ধারণা পায়, ভাবনায় বসে, ধর্মীয় বোধের সন্গে একতা লাভ করতে থাকে ক্রমশ এবং প্রতীকের আলো দুলতত থাকে বস্তু ও কল্পনার চারধারে, তখনই সর্বজনীনতা ও ব্যাপ্তির দিকে এগোয়। মিথের মধ্য একেশ্বর জগতের বিভা পাওয়া যায়, এই কারণেই মিথ প্রাচীন মহাকাব্য, পুরাণ, উপনিষদ ধর্মীয় কাহিনি কিংবদন্তির মধ্যে লুকিয়ে থাকে। মিথের মধ্যে কাহিনি, কাহিনির সংকেত, ঘটনার তাৎপর্য এবং শব্দ ও ছবির প্রতীক মিশে আছে। মিথ পুরানো কাহিনি বলয়কে আমাদের চোখের সামনে স্মরণ করিয়ে দেয়। রামায়ণ, মহাভারত, ক্যান্টারবেরি টেলস, জাতক, আরব্য রজনী এবং কিংবদন্তি ইত্যাদির মধ্যে মিথের প্রভাব লক্ষ্য করা যায়। মানুষের কল্পনা যখন স্বর্গ মর্ত্য পাতাল একসন্গে ঘুরে বেড়ায়, লেক্ষেত্রে দৈবশক্তি ও বিশ্বজনীন ঘটনা মানুযকে বিশাল করে তোলে, মহাকাব্যের মধ্যে এই বিশ্বজাগতিক বোধের অস্তিত্বের জন্যই মিথ লেখানে সার্থক হয়ে ওঠে।

97

Litinfinite Journal is Indexed By MLA Directory Of Periodicals \& MLA International Bibliography, EBSCO, ProQuest, SCILIT, Ulrichsweb \& Ulrich's Periodicals Directory, ICI World Of Journals, J-Gate, JISC-Sherpa Romeo, DRJI, EuroPub \& Other Major Indexing Services. (This Open Access article is published under a Creative Commons Attribution Non-Commercial 4.0 International License.) 
মধুসূদন যেমনভাবে মিথকে ব্যবহার করেছেন, রবীদ্দ্রনাথ আবার সেই পদ্ধতি ব্যবহার করেননি। মধুসূদনের মিথের কাহিনি ও চরিত্রের প্রায় সব কিছু নিয়ে একটি পূর্ণ অবয়ব ছবি গড়ে তুলে গোপনে সংগীতের তাৎপর্য উপমার ছবিতে ফুটিয়ে তুলেছেন। মধুসূদন মেঘনাদের মতো বড়ো মহাকাব্য রচনায় পর শেষে একটি মাত্র বাক্যের পংক্তিতে একটি উৎপ্রেক্ষায় সমগ্র কাব্যের মূল বিষয়বস্তু ও রসানুভূতি চমকিয়ে দিয়েছেন' বিসর্জি প্রতিমা যেন দশমী দিবসে'১০ ( রায়, ২৩ )। প্রতিমা বিসর্জনের কাহিনীর ভেতরে সমগ্র জাতির বেদনা ও অশ্রু, ধর্মীয় ও অনুভূতির গূঢূতা ও বিশ্বাস, ঈশ্বর ও মানুষের সঙ্গে সম্পর্ক, মানুষের মধ্যে দেবতাকে হারাবার কান্না, এই কান্নায় মানুষের অপরাজেয় শক্তি ও আকাজ্খায় বিশ্ববোধ একসঙ্গে উদ্ভাসিত হয়ে ওঠে। রবীন্দ্রনাথ পুঙ্খানুপুঙখ বর্ণনায় যান না, পরিপার্শ্ব অনেক সময় অনুপস্থিত থাকে, একটি মাত্র শব্দের নির্দেশে অনুষঙ্গ জ্বালিয়ে মনোমতো মিথ তৈরি করতে থাকেন।

রবীন্দ্রনাথের পরে অনেকেই মিথের ব্যবহার করেছেন কাব্যে। কিন্তু এই সর্বগ্রাসী ব্যাপকতার ক্ষুধা কাব্যের মধ্যে নেই। রবীন্দ্রনাথ যখন জীবিত ছিলেন তখন দ্বিজেন্দ্রলাল ক্ষীরোদপ্রসাদ মিথ ব্যবহার করেছেন ঠিকই কিন্তু তাঁদের মিথ সর্বজনীনতা পায়নি, পুরাণের মতো প্রাচীন স্তূপ হয়ে আছে। মন্মথ রায় কংসের মধ্যে যুগোপযোগী ভাবনা মেশাতে চেষ্টা করেছেন, যেমন করেছেন যামিনী রায় তাঁর ছবির মধ্যে। সাহিত্যের থেকেও চিত্রে মিথকে তাঁর রোমান্টিকতার রহস্যে আরও পূর্ণ ও উন্নত করে তুলেছেন অবনীন্দ্রনাথ। অবনীন্দ্রনাথ ও নন্দলালের ছবি যদি আমরা অবনীন্দ্রনাথের ছবি যদি আমরা পাশাপাশি রাখি দেখব যে, দুজনের ক্ষেত্রে মিথের ব্যাবহার ভিন্নভাবেই। শিল্পী তুলির অংকন বোধের তীব্রতায় অবনীন্দ্রনাথ স্থূল বস্তকে আগুনের গোপন মধুর আলোয় অদৃশ্য রহস্যে মুহূর্তে রূপান্তরিত করতে পারেন। কিন্তু নন্দলালের আঁকা চিত্রে ফর্মের শিক্ষক জনিত কৃতিত্ব আছে, ছবির স্পষ্টতা ছাড়িয়ে খুব ঊর্ধ্ব আনাবাস উঠতে পারে না। সাহিত্যের মিথকে দেখতে হনে নন্দলালের চিত্রের মত দেখা দরকার।

বন্ধ্যা ও উর্বরা এই মিথকে ভাঙিয়েই এলিয়ট 'পড়োজমি' রচনা করেছেন সমগ্র ইউরোপীয় সমাজ পটভূমিকায়। উর্বরা শক্তির প্রতীকরূপে তাই বৃষ্টি, জল, মাছ, সমুদ্র, নদী আসে; এর বিপরীত ছবিতে ফুটে ওঠে পাথর, মরুভূমি ও শহরের ভিড়। স্যাঁ - জন প্যার্স 'আনাবাস' কাব্যে অভিযানের মধ্য দিয়ে মানুষের অন্তরের অদম্য যাত্রাকেই বিশাল দেশেকালেই মরুভূমির প্রান্তরে পাহাড়ে নদীর শ্রোতের ওপর দিয়ে প্রতীকিত করতে চাইছেন। অশ্বশাবক আমাদের হৃদয়ের, গাছের সবুজ পাতার নিচে যে দাঁড়িয়ে আছে প্রাণের সবুজ 
বেদনার মতো। এরই অন্যরূপ জয়েসের 'ইউলিসিস', পাউন্ডের 'কান্টোজে' এবং পল ভালেরির 'সাপে' অভিশাপে কবিতায়।

বুদ্ধদেব বসু 'তপস্বী ও তরঙ্গিনী' নাটকে ঋষ্যশৃঙ্গের মিথকে প্রেম ও অপ্রেমের মধ্যে নর-নারীর মিলনে ফার্টিলিটি কান্ট বা উর্বরা শক্তিকেই প্রকাশ করতে চেয়েছেন। অনাবৃষ্টি দেশে যে জল নেমে এল ঋষ্যশৃঙ্গের পদার্পণে, সেই জল পৃথিবীকে শস্যপূর্ণ করে তুলেছে জাদুশক্তির একাত্মতায়। আকাশের যে জল নেমে আসে মাটিতে করুণা ধারায়, সেই তৃষ্ণার জলই পতিতার হৃদয়ে প্রেমের মতো বর্ষিত হয়েছে ঋষ্যশৃঙ্গের মুগ্ধ দৃষ্টিতে জাদুর আলোতে। নারী ছাড়া পৃথিবীতে কোনো মানুষের মুক্তির সম্ভাবনা নেই, সংস্পর্শহীন ঋযি নারীর চোখের আলোয় প্রেনের স্পর্রে পূর্ণ হল, এই পূর্ণতার মধ্যে তার সাধনা; যতদিন নারীর হৃদয়ের এই বেদনার গন্ধ পায়নি, ততদিন লে পূর্ণ হয়নি। আসলে, বুদ্ধদেব বসু বৃষ্টিকে এই দুই উপায়ে আলোয় তাৎপর্যমণ্ডিত করেছেন। যদিও মিথের মধ্যে ফ্রুয়েড, এলিয়ট, রবীন্দ্রনাথ ও লরেন্স ওতপ্রোতভাবে জড়িয়ে আছে, বিশেষ করে রবীন্দ্রনাথের 'প্রতিতা'র হুবহু ধ্বনি শোনা যায় পংক্তির মধ্যে, তবু বুদ্ধদেব বসু আধুনিকতার প্রেক্ষাপটকে সঙ্গে নিয়ে তিনি মহাভারতের কাহিনিকে অর্থের তাৎপর্য দিয়েছেন। বুদ্ধদেব বসু এলিয়টের অনুকরণে কোরাস এনে ধর্মীয় বোধের সঙ্গে মিথকে সংযোগ করতে চাইছেন।

আধুনিক বাংলা কবিতায় দেশি ও বিদেশি মিথের ব্যবহার প্রচুর। বিষুণ দে'র কবিতায় মিথের ব্যবহারও এদিকে শীর্ষস্থানীয় ও ঐশ্বর্যময়। এর পরেও মিথের ব্যবহার জীবন দর্শনের তাগিদে কোনো না কোনোভাবে ব্যবহার করেছেন। বাংলায় বহু প্রচলিত মিথ বেহুলা, ঠাকুরমার ঝুলি ইত্যাদি। আমি বিশ্বাস করি, একালের সব কবিই কোনো না কোনো প্রকারে বেহুলাকে মিথের সংকেতরূপে কাজে লাগিয়েছেন কবিতায়। জীবনানন্দ বেহুলাকে দেখেছেন বাংলা প্রকৃতির রূপসৌন্দর্যের স্নিগ্ধ রূপময়তায় বিভাগয় -

" . . . বেহুলাও একদিন গাঙুড়ের জলে ভেলা নিয়ে -কৃষ্ণা দ্বাদশীর জোৎস্না যখন মরিয়া গেছে নদীর চরায় -সোনালি ধানের পাশে অসংখ্য অশ্বথ বট দেখেছিল, হায়, শ্যামার নরম গান শুনেছিল, -- একদিন অমরায় গিয়ে ছিন্ন খঞ্জনার মতো যখন সে নেচেছিল ইন্দ্রের সভায়, বাংলার নদী মাঠ ভাঁটফুল ঘুঙুরের মতো তার কেঁদেছিল পায় "১১ ( রায়, ২৬)। 
জার্মান গীতিনাট্যকার হবাগনের তাঁর কাব্যনাট্য সংগীতের সুরের সাহায্যে সাংকেতিক ভাষায় একটি জাতির মিথের মধ্য দিয়ে একটি দেশের সমগ্র মানুষের আশা-আকাজ্ষাকে রূপায়িত করতে চেয়েছিলেন মঞ্চে। তিনি চেয়েছিলেন মিথের সঙ্গে জাতি বা ফোক এবং শিল্পকে একসঙ্গে সম্পর্কিত করতে। গ্রিক ট্র্যাজেডির ক্ষেত্রে দেখা যায়, গ্রিক মিথের বিষয় ও আত্মিক রূপ শিল্পময় প্রকাশে প্রত্যক্ষ। সেটা চিরকালের জন্য সত্য কেননা এই মিথ হচ্ছে কবির আদর্শ বস্তু। এই বস্তু স্বদেশীয়, একটা জাতির নামহীন কবিতা। উন্নত সংস্কৃতির বিশেষ যুগের মহৎ কবিরা যুগ যুগ ধরে এইসব মিথ নতুনভাবে ব্যবহার করেন। বিশ্বায়ন ও আধুনিকতা প্রেক্ষাপটে সময়ের সঙ্গে সঙ্গে সবকিছু নতুনভাবে ভাবনাচিন্তাকে ত্বরান্বিত করছে। সেগুলি নতুনভাবে আমরা সাহিত্যে ব্যবহার করছি। ইয়েটস যখন আইরিশ লোকসাহিত্যের মধ্যে জাতির প্রাণ স্পন্দন খুঁজতে চান, শুনতে পান, তাকে কাব্যে প্রকাশ করতে চেষ্টা করেন, তখন কবি এই আদিম চিত্রকল্পকেই মিথের মধ্যে এবং লোকসাহিত্যের গল্প কবিতায় ধরতে চেয়েছেন। তিনি তাঁর কবিতায় চিরন্তন সত্য দেখতে পান জাতিগত সত্তা প্রতিষ্ঠার মাধ্যন্ ।

মিথের ব্যবহারে একালের প্রায় সকলেই করেছেন কবিতায়, উপন্যাস এবং নাটকেও। কিন্তু এলিয়েটের মিথেরব্যবহার অন্যদের থেকে স্বতন্ত্র। সেখানে কাহিনি ঘটনা চরিত্রের ঐক্য বা সংলগ্নতা নেই। তিনি যে মিথ ব্যবহার করেন, তার সঙ্গে অনেক মিথ যুক্ত করেন, এতে মিথের কাহিনি আলাদা হয়, বৈচিত্র আলে কিন্তু সব মিথের অন্তর্নিহিত তাৎপর্যকে ঐক্যসূত্রে ইঙ্গিতবহ করে তোলেন। ফলত মনে হয় মিথগুলি ভাঙা, টুকরো, বিচ্ছিন্ন। কিন্তু তিনি ভাঙা বিচ্ছিন্ন চিত্রকল্পগুলিকে সাজিয়ে তার মধ্য থেকে মূল সত্যকে উদ্ধারিতে করতে চেয়েছেন। এলিয়টের পড়োজমির মূল মিথ উদ্ভিদ জীবনের বন্ধ্যা ও উর্বরতার মিথ, যার সাথে অ্যাডোনিস, আত্তিস ও ওসিরিস মিশে গেছে। মৃত্যু ও পুনর্জন্ম মিশে গেছে একসঙ্গে এবং সমস্ত পৃথিবীর মিথ এর মধ্যে যুক্ত, ফলে বিশ্বে উদ্ভিদ জীবনের সর্বজনীনতা লক্ষনীয়। এই উদ্ভিদ জীবনের মিথের সঙ্গে টিরেসিয়াস ও হোলি গ্রেইলের মিথ জুড়ে দেওয়া হয়েছে। দান্তের 'ডিভাইন কনেডি' তে নরকের অন্ধকার থেকে পুনরুজ্জীবনের মধ্য দিয়ে স্বর্গীয় আলোর উত্তরণে কাহিনি। এখানে মৃতের ও পুনরুজ্জীবনের খৃষ্টীয় কাহিনির অনুষঙ্গ আছে। এই বিচিত্রতার জন্যই মিথের ব্যবহার সম্বন্ধে বিভ্রান্তি আমাদের চমকিত করে। বিচিত্র মিথের সঙ্গে তিনি সর্বদাই সক্ষম হয়েছেন একালের মানুযের জীবন ও কাহিনি, বাস্তব শহর ও পরিবেশ ঢুকিয়ে দিতে। এলিয়ট এতোটা জটিলভাবে তাঁর কাব্যে দেখিয়েছেন যা জয়েসও তাঁর 'ইউলিসিস' গ্রন্থে দেখাতে পারেনি কিন্তু 
এলিয়টের কাছে 'ইউলিসিস' আদর্শ হিসেবে উদ্ভাসিত ছিল। যদিও শেলি তাঁর অ্যাডোনিস কাব্যে এতটা জটিলভাবে না হনেও বর্তমানের সঙ্গে গ্রিলের অতীত মিথ ও প্রাচীন কাব্য ইতিহাসকে একসরে যুক্ত করে বৈচিত্র্য ও বিস্তার আনতে পেরেছেন। এখানে এলিয়টের 'পড়োজমি' র সঙ্গে সাদৃশ্যও কম নয়।

এলিয়টের 'পড়োজমি' কাব্যের মিথ বৃষ্টি ও মেঘ। সমস্ত কাব্য গড়ে উঠেছে বৃষ্টি মিথের পরিকল্পনায়। সৃষ্টি ও পুনর্জন্ম ধ্বংসের পর প্রকৃতি ও জীবনে যেমন সত্য, তেমনি জীবন্ত মানব জীবনেও মানুষের হৃদয়ে। ঋতুচটের্রের মতো প্রকৃতির ধ্বংস ও সৃষ্টির লীলা দিনরাত্রির মতো উঠছে নামছে। মানুমের বন্ধ্যা জীবনের সময় বৃষ্টির আবির্ভাব হয়ে পুনর্জন্ম হয় ও সৃষ্টির চিরন্তনতা নিয়ে আলে, সেজন্য পাথুরে পাহাড়ের বুকে জলের ধ্বনি, মরুতূমি বুকের উপর নাইটিংগেলের গান একসঙ্গে শোনাতে চেয়েছেন কবি। এলিয়ট তাঁর 'পড়োজমি' কাব্যে তারপরও সাধনার কথা বলেছেন। আমার পিছনে ককনো পড়োজমি, কিন্তু আমি তীরে বলে মাছ ধরতে চাইছি, মাছের সন্থে উর্বরা সৃষ্টিকে মেলাতত চাইছি। এগুলির জন্য দরকার সাধনা ও মননশীল চিন্তা। এই কবিতার শুরুতেই মিথের আগমন, এই মিথের সঙ্গে ধর্মীয় পুনরুত্জীবনও জড়িত -

April is the Cruellest month, breeding

Lilacs out of the dead land, mixing

Memory and desire, Stirring

Dull roots with spring rain.

অনেকেরই মনে হবে, অ্যাডোনিসের মিথ গ্রীসীয়, তা নয়। এটি ফিনিসীয়। উদ্ডিদ দেবতা মাটির উর্বরা শক্তির সঙ্গে এর যোগ। অ্যাডোনিস তাঁর পূর্বের উদ্ভিদ দেবতা অ্যানেইন ও মোটসকে নির্বাসিত করে দিয়েছেন তাঁর ব্যাপকতায় ও প্রসারে। জানা যায়, গাছ থেকে তাঁর জন্ম এবং তাঁর মা নিজেকে গাছে রূপান্তরিত করে দিয়েছেন। এমনকি অ্যাডোনিস এতটাই সুন্দর যে, তাঁর সৌন্দর্যে আফ্রোদিতে মুগ্ধ। তাঁকে একটি বাক্সে বন্ধ করে রেখে মাটির নিচের দেবী পের্সেফোনের কাছে রেখে দেন, এও শর্ত ছিল পের্সেফোনে খুলে দেখবেনা না বাক্সের ভিতরে কী আছে, কিন্তু কৌতূহলবশত পের্সেফোনে বাক্সটি খুলে দেখে অ্যাডোনিলের সৌন্দর্য মুগ্ধ হয়ে যায়। এবং আফ্রোদিতেকে বাক্সটি দিতে অস্বীকার করেন। এটি শেষ পর্যন্ত দ্বন্দ্বে পৌঁছায়, সমাধানের জন্য দুই দেবী জিউসের কাছে আলেন। জিউসের নির্দেশে ছ- মাস অ্যাডোনিস মাটির উপরে এবং ছ- মাস নিচে থাকবেন। অ্যাডোনিসকে দেখে আফ্রোদিতেও এতটাই বিরহ হয়ে পড়েন শে, শেষ পর্যন্ত তার প্রেনে পড়ে 
যান। অ্যাডোনিস সবথেকে বেশি শিকারে আনন্দ পেতেন, সেজন্য আফ্রোদিতেকে শিকারে যেতে বারণ করেছিলেন কিন্তু শেষ পর্যন্ত শিকার করতে গিয়েই বন্য ভালুক তাঁকে মেরে ফেলে।

বিশ্বসাহিত্যে শ্রেষ্ঠ ট্রাজেডি এই মুহুর্তে বলা যেতে পারে 'ওইদিপৌস' কে। থিবসে ওইদিপৌসের রাজত্বে সারা দেশে মহামারী, মৃত্যু, শুষ্কতা, প্রাণহীন বন্ধ্যা জীবন, শস্য হয় না। নারী গর্ভধারণে ব্যর্থ, বৃষ্টি নেই, চারিদিকে শুধু মৃত্যু ছড়িয়ে আছে। ক্ষুব্ধ ঝড়, আন্দোলিত শহর। শহরকে শূন্য করে দেবার জন্য জ্বরের বিবর্ণ দেবতা সমস্ত মানুষের উপর মূর্ছার মতন ছড়ানো। যিনি অন্ধকার পাতালের দেবতা এই মহামারীর প্রাক্কালে তিনি যন্ত্রণায় হাহাকার করছেন এবং বিলাপ করে চারিদিকে ঘুরে বেড়াচ্ছেন। কিন্তু জীবনের ধাঁধার রহস্য কোথায় শেষ হবে, স্বর্গের গোপন পথ কোথায় ? সমস্ত থিবস আজ মরুভূমি, কোথাও প্রাণের স্পর্শ নেই। এসবেরই কোনো সমাধান নেই, উত্তর নেই । মানুষের ভালোবাসা, স্নেহ কিছুই নেই, আছে শুধু অন্তহীন জিজ্ঞাসা।

ওইদিপৌস নিজের পাপেই থিবস প্রদেশকে মরুভূমি তৈরি করেছে, মায়ের সঙ্গে স্বামী হিসেবে সহবাসে, মাতার গর্ভে নিজের সন্তানের উৎপাদনে, নিজের পিতাকে হত্যা। ওইদিপৌস যে দেশে জন্মগ্রহণ করে সে দেশ থেকে নির্বাসিত, নিজের দেশে ফিরে এসেছে অজানা পরিচয় ও রহস্য অন্ধকার নিয়ে, পিতৃহত্যার পাপে নিজের হাতকে কলুষিত করেছে এবং নিজের শরীর ও আত্মা করেছে নিজের মায়ের সঙ্গে যৌনসঙ্গমে। নিজের ভাই ও ভগিনী হয়েছে পুত্রকন্যা। ওইদিপৌসে ঔদ্ধত্য ও অহংকার বিশ্বনিয়নের কাছ থেকে এসে দূরে সরিয়ে निয়ে গেছে।

তাইরেসিয়াস এই দৃশ্য দেখেছেন, জেনেছেন, কিন্তু এই মৃত্যু ও পতন থেকে বাঁচাবার পথ তাঁর জানা ছিল না। তাই তাঁর জ্ঞান ভারি বোঝার মতো কেননা এই জ্ঞান কোনো কাজে আসে না। অথচ জোর করা হচ্ছে ওইদিপৌস এর জ্ঞানকে প্রকাশ করবার জন্য কারণ বিশ্বের আঁধার উন্মোচিত হয়ে ওঠে। তাতে মানুষ ও জগৎ অভিশপ্ত মনে হয়। এই অভিশাপ থেকে মুক্তির পথ নেই। এই অভিশাপ থেকে মুক্তির জন্য ঈশ্বরের কাছে প্রার্থনা করেছেন। গোটা থিবসবাসীকে ওইদিপৌস পাপ ও অপরাধেই দূষিত করেছে।

আন্তিগোনে নাটকেও তাইরেসিয়াস সেই একই কথা বলেছেন ক্রেওনকে। মানুমের ঔদ্ধত্য ও একগুঁয়েমি, অহংকার মানুষের বোধ ও ধারণাকে হত্যা করে। সেই পথ দিয়েই মানুষের ধ্বংস আছে, তাই মানুষকে বিনম্র 
হতে হবে, শ্রদ্ধা করতে শিখতে হবে। এই কারণেই মানুষের ধারণা ও বোধকে প্রসারিত করা আবশ্যক বলে আমার মনে হয়। মানুষের ভুলের শাস্তি দেবার জন্য ভগবানের ধ্বংসকারী হাত দ্রুত নেনে আসে।সেজন্য ক্রেওন বলেন -- আমি মনে মনে ভয় পাই ; এটাই সবচেয়ে শ্রেষ্ঠ উপায় একজনের জীবনের মধ্য দিয়ে দেখা যে নিয়ম প্রতিষ্ঠিত আছে ; এই নিয়মকে লঙ্ঘন করতে পারে না; ওইদিপৌস ও ক্রেওন তাঁদের জীবনে এই নিয়ম লঙ্ঘন করেছিলেন বলেই, তাঁদের জীবন অভিশপ্ত, তাঁদের বেঁচে থাকার মধ্যেই ধ্বংস নেমে এসেছে। আন্তিগোনে নাটক কোরাসের মতোই, তাইরেসিয়াসের বাণীর মতোই, এলিয়টও 'পড়োজমি'- র মধ্যে মুক্তির পথের উপায় দেখেছেন মানুষের হৃদয়ের সংযম, দয়ারমধ্যে, উপনিষদের উক্তির মধ্যে। এখানেই গ্রিক ও ভারতীয় সভ্যতা ও সংস্কৃতির মিলন।

' আন্তিগোনে ' এবং ' রাজা ওইদিপাউস ' নাটকে তাইরেসিয়াস অন্ধ ভবিষ্যদ্বক্তা, তিনি অন্তরের আলো জ্বেলে সবকিছু দেখেন, জানেন, বোঝেন। তাঁর বোধে তিনকাল এক হয়ে গেছে। তিনি কোনো দেশের রাজার অধীন নয়, অ্যাপোলো ও জিউসের ভক্ত, স্বাধীন। আরেকটি কাহিনিতে আছে তাইরেসিয়াস একদিন দুটি সাপের সঙ্গম দেখতে পান এবং লাঠি দিয়ে তা প্রহার করার সঙ্গে সঙ্গে তিনি নারী হয়ে যান। এই বিশ্বাস তাদের ছিল এবং আস্তে আস্তে মিথ বিশ্বাসে পরিণত হয়। যদিও বর্তমান সময়ে কিছুটা হুেেও হ্রাস পেয়েছে। তাইরেসিয়াস কিছুদিন পরে একই দৃশ্য দেখতে পান, তিনি এবার পুরুতে রূপান্তরিত হন। সুতরাং তাঁর দেহে একই সঙ্গে নারী ও পুরুষ বিরাজ করছে, তাদের দুজনেরই অভিজ্ঞতা তার শরীরে। শক্তি ও শক্তিমান একাত্ম। জিউজ ও হেরার মধ্য বিবাদ বাধে, যৌনসঙ্গমে নারী ও পুরুযের মধ্যে কে সবচেয়ে আনন্দ পায়, তাই রেসিয়াসকে মধ্যস্থতা করতে বললে তিনি বলেন, নারী যৌনসঙ্গনে বেশি আনন্দ পায়। এই কথা শুনে রাগে ও ক্রোধে হেরা তাঁকে অন্ধ করে দেন। ক্ষতিপূরণ স্বরূপ জিউস তাঁকে দীর্ঘ জীবন ও ভবিষ্যদ্বাণীর শক্তি সমর্পণ করেন।

মিথলজি এবং ফোক কোনো দেশের শিকড়কে বুঝতে সাহায্য করে। মানুলের মধ্যে লোকসংস্কার, লোকবিশ্বাস ইত্যাদি যখন অনেকদিন ধরে জন্মায় তা একটা সময় মিথ বিশ্বাসে পরিণত হয়। এই বিশ্বাস কখনো স্থায়ী নয়, পরিবর্তনশীল। বাংলার পাশাপাশি যদি প্রতিবেশি রাজ্যের দিকে তাকাই দেখবো যে, শিব বিহারের সর্বাধিক পূজিত দেবতা। বিহারে ছট পূজা প্রধান ও অত্যন্ত জনপ্রিয় লোক উৎসব। পৌরাণিক ও লোকতাত্ত্বিক ব্যাখ্যানুযায়ী ছট পরব বর্তমানে আধুনিকতার আচ্ছাদনে পালিত হয়। ছট পরব অর্থাৎ সূর্যপূজার ধারাও 
সুপ্রাচীন। লোকবিশ্বাস হল যে, ছট পরবে সূর্য পূজার রবিবার উপবাসে থাকা, হোম করা এবং লবন ছাড়া খাদ্য গ্রহণ করলে পাপমুক্তি ঘটে। কিংবদন্তি অনুসারে ছট ব্রত দেবী পালন করেছিলেন, যার সাক্ষী ছিলেন সূর্যদীপ। তাই সূর্য পূজার অনুষঙ্গে দেবী ভগবতী বা ছঠী মাতার পূজাও প্রচলিত। ষষ্ঠী তিথিতে এই পরব অনুষ্ঠিত হয় বলে দেবী ভগবতীকে ষষ্ঠীমাতা বা ষষ্ঠীদেবী রূপে গণ্য করা হয়ে থাকে। কারণ পুত্র কামনা এই ব্রতের অন্যতম উদ্দেশ্য। ষষ্ঠী ব্রত বা সূর্য পূজা রাজস্থান, গুজরাট এবং মহারাট্ট্রের 'মাঘ সপ্তমী', 'রথ সপ্তমী', 'অচলা সপ্তমী' এবং 'পুত্র সপ্তমী' নামে অভিহিত। পশ্চিমবঙ্গে এই সূর্যপূজা 'ইতুব্রত' নামে পরিচিত। রমনীরা এই ব্রতের আয়োজন করার উদ্দেশ্য হল, পুত্র সন্তানের মঙ্গল কামনা, পুত্র লাভ, ধনধান্য ও সুখ-সমৃদ্ধিলাভ। মূলত এটি বিহারের প্রধান উৎসব। এছাড়াও বিহারের শিব পূজা, শীতলা পূজা, মাঘ মন্ডলের ব্রত, জিওডিয়া, বারহমাসা ইত্যাদি উৎসব লক্ষ্য করা যায়।

হিমালয় ভারতীয় সংস্কৃতির প্রতিভূ। এই পর্বতমালার জনগোষ্ঠীর লোকসাহিত্য ও সংস্কৃতি নিঃসন্দেহে গরিমাময়। ভারতের অন্যতম পার্বত্য রাজ্য হল হিমাচল প্রদেশ। হিমালয়ে বহু জাতির সংমিশ্রণে এক সমুন্নত লোকসাহিত্য ও সংস্কৃতি গড়ে উঠেছে। তবে একথা সত্য যে, হিমালয় মূলত শিবেরই আলয়। সেজন্য এই রাজ্যের হিন্দুদের প্রধান উপাস্য হলেন শিব। এখানে বিভিন্ন লোকদেবদেবী দেখা যায় - মহাসু, মহিমহেশ, বুলডাং, বৈজনাথ প্রভৃতি। ওখানকার অধিবাসীরা শিবের পরেই শক্তিপূজা প্রাচীনকাল থেকে করে আসছে। শিবকে নিয়ে তাদের বিশ্বাস, সংস্কৃতি সবই গড়ে উঠেছে। এই বিশ্বাস তাদের জীবনে মিথে পরিণত হয়েছে। এছাড়াও এই রাজ্যে বিভিন্ন উপজাতির বসবাস এবং তাদের নিজস্ব দেবদেবী রয়েছে যেমন গদ্দী উপজাতিদের প্রধান দেবতা - কৈলংগ, কুল উপত্যকার মলানা গ্রামের একছত্র দেবতা হলেন - জামলু। পাঙ্গীর উপজাতিদের জাগ্রত দেবী হলেন - মিংঘল, মন্ডী জেলার চচ্যোট মহকুমা অধিবাসীদের পূজিত দেবতা হলেন - কামরু নাগ, কুলুর ভহয়াত অঞ্চলের প্রসিদ্ধ দেবতা হলেন নাগ মণঢোর।

' হাঁসুলী বাঁকের উপকথা ' র কাহারেরা মিথের মধ্যে বসবাস করে। তাদের দৈনन্দিন জীবনের প্রতিটি মুহূর্ত বাবা ঠাকুরের মিথ দ্বারা নিয়ন্ত্রিত। সেই মিথ ক্রমে ভেঙে পড়তে শুরু করে। উপন্যাসের শুরুতেই করালী বাবাঠাকুরের বাহন চন্দ্রবোড়া সাপটি মেরে ফেলার ঘটনার মধ্য দিয়ে মিথ ভাঙবের ধারা লক্ষ্য করা যায়। আলব্যের কাম্যুর বিখ্যাত উপন্যাস ' মিথ অফ দি সিসিফাস ' -এ দেবতারা সিসিফাসকে চিরকাল একটা পাথর পাহাড়ের চূড়ায় উঠানোর শাস্তি দেন, যেখান থেকে পাথরটা নিজের ভরেই আবার নিচে পড়ে যাবে। এখানে 
গ্রিক পুরাণের গল্প দেখা যায়। হোমারের ওডিসিতে আছে, ওভিদে আছে। জিউসের চেয়ে নিজেকে বেশি চালাক ভেবে সিসিফাস চালাকি করতে গিয়েছিল। তাই তার জীবনে অভিশপ্তে পরিণত হয়। কাম্যু কী চমৎকারভাবে গ্রিক পুরাণ কাহিনি নিয়ে পুরানো এবং আধুনিকতার সঙ্গে নতুন কাহিনির ব্যাখ্যা করলেন। লেখক বলেছেন, সিসিফাস যখন পাথর ঠেলে তোলে, তখন সে হাসিমুখে সেই কাজটা করে, একটু আগে কী ঘটেছিল, একটু পরে কী ঘটবে, এটা তার ভাবনাতে নেই ।

পাঠক মাত্রেই বুঝতে পারেন সিসিফাস আমাদের অর্থহীন নায়ক। তার আশক্তি আর নির্যাতনের মধ্য দিয়ে তিনি নায়ক। দেবতাদের প্রতি তার অবজ্ঞা, তার মৃত্যুঘৃণা, তার জীবনের আসক্তি, তার অবর্ণনীয় অর্থহীন শাস্তির কারণ হয়েছিল। প্রবন্ধে আলোচনায় ' হাঁসুলী বাঁকের উপকথা ' তে যে প্রেক্ষাপট রচিত হয়েছে সেখানে একরকম ভাবে লোকজীবন ও মিথ চিত্রায়িত করেছেন। আবার পাশাপাশি দেশি ও বিদেশি সাহিত্যের আলোচনাতে আরএকরকম ভাবে মিথের ভাঙা গড়ার করার প্রক্রিয়া দেখা যায় সময়ের সঙ্গে সঙ্গে পরিবর্তন হতে। এখানেও নতুন নতুন বর্গের ( Genre ) সূত্রপাত ঘটছে কেননা ঔপন্যাসিক তারাশঙ্কর বন্দ্যোপাধ্যায় একরকম ভাবে মিথের উপস্থাপনা ফুটিয়ে তুলেছেন সেখান থেকে আবার রবীন্দ্রনাথ, আরব্যরজনী, মাইকেল মধুসূদন, শেক্সপিয়র, মিল্টন, এলিয়ট ইত্যাদি এঁরা মিথকে নানারকম ভাবে বাস্তবায়িত করেছেন। এবং এঁরা প্রত্যেকেই নিজস্ব ঘটনায় স্বতন্ত্র। মিথের সঙ্গে তো মানুষের লোকসংস্কৃতি, বিশ্বাস, আচার-ব্যবহার সবই অন্তর্ভূক্ত। এঁদের মিথ থাকছে কিন্তু সময়ের প্রেক্ষাপট অনুযায়ী বদলে যাচ্ছে। আরব্যরজনী, ক্যান্টারবেরি টেলস, ডেকামেরনে বিভিন্ন কথক বিভিন্ন ভাবেই গল্প বলছে, আখ্যান কৌশল ( Narrative Technic) তৈরি হচ্ছে। এখানেও রোলাঁ বার্তির ' অন্তরপাঠশৃঙখল ' ( Intertextuality )এরকথা বলা যেতে পারে কেননা একটা পাঠ ( Text ) তৈরি হয় অগণিত পাঠের সহযোগে এবং বহু পাঠসংস্কৃতি থেকে। কোনো পাঠ নির্দিষ্ট এবং স্বয়ংসম্পূর্ণ নয়। পাঠে ( Text ) আধুনিকতার প্রভাব পড়ায় বিশ্বাস, সংস্কৃতি বদলে গিয়ে মিথের বহুকৌণিক কণ্ঠস্বরের সমাহার ঘটেছে। প্রাসঙ্গিকভাবে বাখতিনের ' ডায়ালজিক ইমাজিনেশন ' বা ' সাংলাপিক কল্পনার ' কথা। মানুযে মানুষে কীভাবে আদান-প্রদান হয়, একজন মানুষ বা মানুষগোষ্ঠী কীভাবে নিজেদের ভাবনা, বেদনা, আবেগ জানাতে পারে তারই তত্ত্ব এই সংলাপবাদ বা ডায়ালোজিজম। এই পৃথিবীতে মানুষের কোনো উচ্চারণ নি:সঙ্গ নয়, পূর্বাপরহীন নয়। 
অভিজিৎ সেন তাঁর 'মিথ ও লোকাচার সম্ভাবনা' প্রবন্ধে তিনি বলেছেন -- 'সাধারণ ভাবে বলতে গেলে মিথ বা লোককথার বহুমাত্রিক ব্যবহার আমাদের সাহিত্যে এখনো খুবই নতুন। সত্তর দশকের ' '্রামে চলো' ব্যাপারটা অন্য অনেক কিছুর মঢো শিল্প-সাহিত্যেও একটা ওলট পালট করেছে মনে হয়।. . . ফলে অন্য মাধ্যমগুলোর মতো গল্প-উপন্যাসেও একটা অবলম্বন আমরা খুঁজতে দেখেছি যা ঠিক ইউরোপীয় নয়'। উপন্যাস, কবিতার ভিতর দিয়ে তৈরি হয় নতুন মিথ, যা গড়ে উঠতে সাহায্য করে তৃতীয় বিশ্বের ঔপনিবেশিক বাস্তবতা। লেখকে মিথকে তৈরি করেন অপর একটি মিথকে সূত্র হিলেবে ব্যবহার করে। আর এই দুটির মিথের মধ্যে যোগলেতু গড়ে তোলার জন্য লেখক জাদুবাস্তবতার প্রয়োগ ঘটান। যেমন দেখা যায়, লাতিন আরেরিকার নোবেল বিজয়ী মার্কেসের ' নিঃসঙ্গতার একশ বছর ' উপন্যালে অনৌকিক বিশ্বাসের মাধ্যমে মিথের সামান্য প্রত্যোগ ঘটান এবং জাদু বাস্তবতার নতুন দিগন্ত রেখা অঙ্কন করলেন। অভিজিৎ লেনের 'রহু চঞ্ঞলের হাড়! উপন্যালের পুরো কাহিনিটিই এমনভাবে বর্ণিত হয়, যেন কোনো রূপকথা বা লোককথার গল্প। কাহিনিটি ফরু হয় লুবিনির জবানিত, তখন শ্রোতা ছিল তার নাতি শারিবা। লুবিনির কাহিনিত ক্রনে কিংবদন্তি ও ইতিহাস মিশে যেতে থাকে। রহু আর দনুর সঙ্গে দেখা হয়ে যায় পীতেম ও জামিরের। কিংবদন্তি চরিত্রের সন্গে ইতিহালের চরিত্রদের। নবজাত শিফুকে দেখে পীতেম যখন বনে ওঠে 'রহু রহু', তখন ইতিহাস কিংবদন্তি হয়ে উঠতে থাকে। এক্ষেত্রে বাখতিনের 'পলিফোনি' র রূপ লক্ষ্য করা যায়। 'পলি' অর্থাৎ 'বহু', 'ফোনি' র উৎসে আছে 'ফোন' অর্থাৎ 'ধ্বনি' বা 'স্বর'। অর্থাৎ উপন্যাস হল একটি বহু স্বরসঞ্গত্মুলক শিল্প -- যেখানে বহু স্বাধীন ও অমিশ্রিত কন্থস্বরের সমাহার ঘটে এবং উপন্যাসে প্রান্তিক মানুষের কণ্ঠস্বরই এক একটি স্বয়ংসম্পূর্ণ অংশ।

মিথ অর্থাৎ ঘনীভূত অস্তিত্বের সন্ধান, জীবনের বিভিন্ন স্তরে উপনীত হওয়ার প্রয়াস, নানানা গ্রন্থিতে জনজীবনকে বাঁধা, দুই বিপরীতাছক তন্ত্রের মধ্যস্থতা করার প্রচেষ্ঠা। এভাবে হয়তো বিভিন্ন স্থানে বিভিন্ন কাজের মধ্যে নতুন এক শ্রেণির মিথ আবিস্কৃত হবে যা জীবনকে পুনরুত্জীবিত করবে। মিথ কী ব্যাপার, কোথায় অবস্থিত, মানব জীবনের মানচিত্রে মোটামুটি কোথায় তার স্থান। এইসব নিয়ে নানান লোক নানান ভঙ্গিতে আঁকতে ভালোবালেন। এখানে যা আঁকা হল এটাও একটা ধরণ। যাঁদের পছন্দ হনো না তাঁরা নিশ্চয়ই অন্যভাবে এঁকে দেখাবেন । কিন্তু ভাবতে ভালো লাগে যে, তা সত্ত্বও একই পৃথিবীর মানচিত্র আমরা নানান রীতিতে আঁকছি। এই যে, ভাবতে ভালো লাগে এর মানে কি এই যে আমি নিছকই 'পৃথিবীটা এর' এরকম একটা দুর্মর মিথের শিকার? প্রশ্ন উঠে, পৃথিবী কী তাহলে এক নয় ? নানা মুনির নানা মত হলেই কী জগৎ 
לুকরো לুকরো হয়ে যায়? এত বড় অয়োক্তিক কথা বলারও যুক্তি কেউ কেউ দেয় যা তা জানি। কিন্তু যাঁরা এসব বলেন তাঁরা কী কখনো ভেবে দেখেছেন , যুক্তি নিজেই একটা মিথ কিনা ?

\section{সূত্রনির্দেশ (References in Bengali)}

“তারাশঙ্কর সংখ্যা ”। কোরক সাহিত্য পত্রিকা ।কলকাতা বইনেলা ২০১৮, মুদ্রণ।

বন্দ্যোপাধ্যায়, তারাশঙ্কর। হাঁসুলী বাঁকের উপকথা । কলকাতা: বেঙ্গল পাবলিশার্স, ১৩৫৪। মুদ্রণ।

মুখোপাধ্যায়, মানবেন্দ্র। উপন্যালের যৎকিঞ্চিৎ । কলকাতা: বঙ্গীয় সাহিত্য সংসদ, ২০১০। মুদ্রণ।

সিংহ, উদয় নারায়ণ এবং অঞ্জন সেন (সম্পাদনা। মিথ সাহিত্য সংস্কৃতি । কলকাতা: গ্রন্থালয় পাবলিশার্স, ১৯৯১।

রায়, বার্ণিক। কবিতার মিথ। কলকাতা: পুস্তক বিপণি, ১৯৮৮-। মুদ্রণ।

\section{References}

Bandyopadhyay, Tarashankar. Legend of the palm bend. Kolkata: Bengal Publishers, 1354, Print.

Mukherjee, Manabendra. As little as the novel. Kolkata: Bangiya Sahitya Sangsad, 2010. Print.

Bandyopadhyay, Tarashankar.Legend of the palm bend. Kolkata : Bengal Publishers , 1354 , Print.

Singh, Uday Narayan and Anjan Sen (edited). Myth Literary Culture. Kolkata: Granthalaya Publishers, 1991. Print.

Roy, Bernick. The myth of poetry. Kolkata: PustokBiponi, 1988. Print.

“The number of Tarasankar”. Korak Sahitya Patrika. Kolkata Book Fair 2018, Print .

Samaresh Mondal is persuring his Ph.D from Indian Comparative Literature Department, Assam University. He has completed his M.Phil in Comparative Literature from the Centre of Comparitive Literature, Visva-Bharati University, West Bengal, India. 